DOI 10. 18307/2020. 0603

(c) 2020 by Journal of Lake Sciences

\title{
太湖梅梁湾藻华暴发一消退周期表层水体溶解性有机质分子特征”
}

\author{
庞佳 ${ }^{1}{ }^{1}$, 许燕红 ${ }^{1}$, 何毓新 ${ }^{1 * *}$, 史 权 $^{2}$, 何 $丁^{1}$, 孙永革 ${ }^{1}$ \\ (1: 浙江大学地球科学学院,杭州 310027) \\ (2: 中国石油大学 (北京) 重质油国家重点实验室,北京 102249)
}

\begin{abstract}
摘 要: 人类活动引起的富营养化对太湖的碳循环模式可能产生严重影响,精细描述太湖藻华暴发一消退周期的溶解性 有机质分子是了解太湖碳库动态变化的关键. 本研究利用傅立叶变换离子回旋共振质谱技术, 以太湖北部梅梁湾 2017 年 5 月至 2018 年 5 月的表层水体为研究对象, 解析藻华暴发一消退周期溶解性有机质的来源和分子组成特征, 进而理解浮 游澡类异常增殖对水体溶解性有机质的影响及其在区域碳循环中的角色. 研究结果表明, 藻华暴发期浮游藻类生产力显 著增加, 使得表层水体的溶解性有机质从含量到分子组成均发生剧烈改变. 含量上表现为溶解性有机碳浓度升高, 分子 组成上表现为 $\mathrm{CHO}$ 类化合物和以脂肪族类化合物为代表的活性组分占比增加, 特征化合物以相对高饱和度和高含氧的 小质量数分子为主. 而在藻华消退期, 随着藻类有机质贡献的减少和有机质降解过程的持续进行, 含量上表现为溶解性 有机碳浓度下降, 分子组成上表现为 CHOS、CHONS 类化合物和富羧酸脂环类化合物等惰性分子占比增加,特征化合物以 大质量数分子和相对低饱和度和低含氧的小质量数分子为主. 研究结果表明, 太湖水体的溶解性有机质分子组成在藻华 暴发期受藻类有机质输入控制, 在消退期受藻类有机质降解的影响.
\end{abstract}

关键词: 富营养化;太湖;梅梁湾;溶解性有机质;分子组成;傅立叶变换离子回旋共振质谱;碳循环

\section{Molecular characteristics of surface dissolved organic matter in Meiliang Bay of Lake Taihu over the algal blooming-disappearance cycle *}

\author{
PANG Jiali ${ }^{1}$, XU Yanhong ${ }^{1}$, HE Yuxin ${ }^{1 * *}$, SHI Quan ${ }^{2}$, HE Ding $^{1} \&$ SUN Yongge ${ }^{1}$ \\ (1: School of Earth Sciences, Zhejiang University, Hangzhou 310027, P.R.China) \\ (2: State Key Laboratory of Heavy Oil Processing, China University of Petroleum, Beijing 102249, P.R.China)
}

\begin{abstract}
Eutrophication induced by human activities may has a critical impact on the carbon cycle of Lake Taihu. Assisted by the technique of Fourier transform ion cyclotron resonance mass spectrometry (FT-ICR MS), we investigated the molecular characteristics of dissolved organic matter in Meiliang Bay of Lake Taihu over the algal blooming-disappearance cycle at 2017-2018 to better understand the influence of algae blooming to the dissolved organic matter pool. The results show that during the algal-blooming period, the massively increasing productivity from algae have changed the amount and the molecular characteristics of dissolved organic matter in water from Lake Taihu. Basically, the concentration of dissolved organic carbon as well as the proportion of the relatively labile components ( $\mathrm{CHO}$-compounds, aliphatic compounds, and low-molecular-mass compounds with higher $\mathrm{H} / \mathrm{C}$ and $\mathrm{O} / \mathrm{C}$ values) increased during the algal-blooming period. While in the algal-disappearance period, due to both the low input of algal biomass and the continuous degradative progress of dissolved organic matter in the water column, the concentration of dissolved organic carbon decreased. Synchronously, the proportion of the relatively refractory components (CHOS-compounds, CHONS-compounds, CRAM compounds, high-molecular-mass compounds, and low-molecular-mass compounds with lower $\mathrm{H} / \mathrm{C}$ and $\mathrm{O} / \mathrm{C}$ values) increased. The dynamics of the molecular characteristics of dissolved organic matter in Meiliang Bay of Lake Taihu indeed propose the critical impact of eutrophication on the regional carbon cycle, asking for further research.
\end{abstract}

Keywords: Eutrophication; Lake Taihu; Meiliang Bay; dissolved organic matter (DOM); molecular characterization; FT-ICR MS; carbon cycle

* 2019-12-31 收稿; 2020-03-31 收修改稿.

国家自然科学基金项目 (41877332) 和中国科协青年托举工程 (2018QNRC001) 联合资助.

** 通信作者;E-mail: yxhe@ zju.edu.cn. 
工业革命以来, 由于人类活动的加剧, 导致温室气体浓度迅速上升, 引发了一系列的气候和环境问题, 全球碳循环受到了世界各国政府和学术界的广泛关注 ${ }^{[1]}$. 内陆水体虽然只占据不到 $1 \%$ 的全球面积,但其在 全球有机碳的运输、转化和储存过程中具有重要的作用 ${ }^{[2]}$. 内陆水体每年从陆地接收 $2.9 \times 10^{15} \mathrm{~g}$ 碳, 其中约 有 $0.6 \times 10^{15} \mathrm{~g}$ 碳被埋藏在内陆水体系统, $1.4 \times 10^{15} \mathrm{~g}$ 碳是以气体形式释放到大气, 而 $0.9 \times 10^{15} \mathrm{~g}$ 碳通过径流被 输送到海洋 ${ }^{[3]}$. 湖泊作为重要的内陆水体要素, 在全球碳循环中也扮演着重要的角色. 据统计, 湖泊每年的 碳埋藏量约相当于海洋碳埋藏量的 $25 \% \sim 58 \%{ }^{[4]}$, 湖泊每年的 $\mathrm{CO}_{2}$ 排放量约相当于陆地河流向海洋输人碳 的 $50 \%{ }^{[5]}$. 近年来, 工农业活动和城市化导致了区域的点源和面源污染, 加剧了湖泊富营养化, 这不仅对湖 泊生态系统造成了严重破坏 ${ }^{[6-7]}$, 也改变了湖泊的碳循环模式 ${ }^{[8]}$. 湖泊藻华暴发一消退的周期过程影响着湖 泊有机碳循环和碳源一碳汇结构 ${ }^{[9]}$. 首先, 湖泊藻华暴发浮游藻类生产力显著增加, 直接导致沉积物中碳埋 藏有机碳增加 ${ }^{[8]}$. 澡华消退之后, 部分有机质进一步选择性的转化和降解并以二氧化碳和甲烷的形式释放 回大气, 而另一部分以相对惰性有机质的形式保存在水体和沉积物之中. 因此, 研究富营养化湖泊的碳循环 动态变化, 明晰其有机碳收支和碳源一碳汇结构, 具有重要的理论和现实意义 ${ }^{[10]}$.

溶解性有机质 (dissolved organic matter, DOM) 通常是指可以通过 $0.45 \mu \mathrm{m}$ 滤膜的有机质, 是水体有机碳 循环动态过程的最直接参与者 ${ }^{[1-115]}$. DOM 来源于生物分泌物和残体碎屑 ${ }^{[16]}$, 是由糖类、蛋白质类、脂类、木 质素、腐殖酸等化合物组成的复杂混合体 ${ }^{[17]}$, 其浓度和分子组成受复杂的生物反应和非生物反应的影 响 ${ }^{[18-20]}$. 因此, 不同分子组成的 DOM 有着不同的生物可利用性和矿化稳定性, 也具有不同的生物地球化学 过程和归宿 ${ }^{[21-23]}$. 精细描述湖泊富营养化背景下藻华暴发一消退周期过程的 DOM 分子特征演化, 可以更好 地理解富营养化背景下湖泊碳库的动态变化.

DOM 分子组成的表征, 重点在于利用谱学技术对 DOM 的化学键、官能团及分子空间构型进行刻画. 傅 立叶变换离子回旋共振质谱 (Fourier transform ion cyclotron resonance mass spectrometry, FT-ICR MS) 可从复 杂 DOM 混合物中解析出数千种精确分子式, 从而可以对 DOM 分子组成进行定性 (化学活性) 和半定量 (相 对浓度) 判定 ${ }^{[24-25]}$. 范氏图 (氧碳原子数量比值 $\mathrm{O} / \mathrm{C}$ 为 $x$ 轴, 氢碳原子数量比值 $\mathrm{H} / \mathrm{C}$ 为 $y$ 轴) 和 Kendrick 质 量偏差图 (Kendrick 质量为 $x$ 轴, Kendrick 质量偏差为 $y$ 轴) 等是使用 FT-ICR MS 数据来定性评价不同类别 DOM 分子组成差异的常用工具 ${ }^{[26]}$. 不仅如此, 研究者们已经建立了一系列划分不同 DOM 分子类别的标 准 ${ }^{[27-29]}$. 该技术已经广泛应用于海洋、河流、湖泊等环境介质 DOM 分子转化的表征及影响因素的 解析 ${ }^{[14,17,27,30-33]}$.

太湖是我国第三大淡水湖泊 (水域面积 $2338 \mathrm{~km}^{2}$, 平均水深 $1.9 \mathrm{~m}$ ), 兼具有调蓄、灌溉、航运、旅游和养 殖等功能 ${ }^{[34]}$. 随着城市化水平的发展, 太湖成为了我国东部富营养化最严重的湖泊之一 ${ }^{[35]}$. 太湖蓝藻水华 最早可以追溯到 20 世纪 50 年代, 太湖五里湖区夏季出现了大量蓝藻、隐藻、硅藻和绿藻并存的现象 ${ }^{[36]}$. 此 后, 藻华逐渐向梅梁湾和竺山湾等区域扩展, 持续时间延长, 蓝藻开始占绝对优势 ${ }^{[37]} .2007$ 年太湖藻华尤为 严重,引发了无锡市饮用水危机, 各级政府加大了对太湖的治理力度, 包括控源截污、内源清淤、引水冲调、 水生态修复等 ${ }^{[38]}$. 然而, 2017 年太湖又一次出现大面积的藻华, 远超过 2009-2016 年的平均水平, 表现为 藻华首次暴发时间总体提前约 30 天 $^{[39]}$, 全年藻华次数和平均面积升高 ${ }^{[40]}$. 其主要原因可能是,在营养盐充 足的背景下, 温度升高、风速较低、日照时间增长均会导致每年藻华首次暴发时间提前和暴发持续时间的延

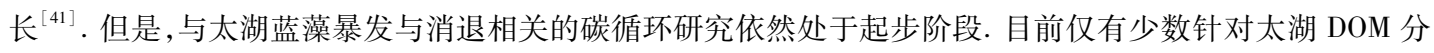
子组成的动态变化研究, 例如, 在对比藻华暴发期与消退期 DOM 分子组成和结构方面, Zhang 等 $^{[9]}$ 通过 FTICR MS 和核磁共振光谱技术, 分析检测了 2007 年 6 月和 2007 年 11 月梅梁湾一个点位的 DOM 分子组成, 发现从 2007 年 6 月到 2007 年 11 月, DOM 中的小质量数分子和 CHON 类分子比例增加, 分子多样性提高. 在分析藻源 DOM 的生物可利用性方面, Bai 等 ${ }^{[42]}$ 通过四级推流式生物膜反应装置, 验证了 DOM 生物可利 用性与微生物群落特征间的关系, 发现藻源 DOM 中活性/半活性分子可占总有机分子的 79\%, DOM 的分子 活性与分解细菌群落多样性呈反比. 在分析浮游藻类对水体 DOM 贡献方面, Liu 等 ${ }^{[43]}$ 通过 FT-ICR MS 和苂 光技术, 对太湖不同湖区的水体 DOM 和藻源 DOM 进行了对比分析, 证实了藻类和水体 DOM 的关联性.

本研究进一步以太湖藻华暴发严重的梅梁湾为研究区域,采集 2017-2018 年藻华暴发一消退周期的表 层水体, 通过 FT-ICR MS 技术, 解析藻华暴发一消退周期 DOM 的分子变化特征, 进而理解浮游藻类对水体 
DOM 的影响及其在区域碳循环中的角色.

\section{1 材料与方法}

本研究于 2017 年 5 月、 6 月、 8 月、 11 月以及 2018 年 1 月、 3 月、 5 月在太湖梅梁湾 $\left(31^{\circ} 25^{\prime} 27.21^{\prime \prime} \mathrm{N}\right.$; $120^{\circ} 11^{\prime} 17.58^{\prime \prime} \mathrm{E}$ ) 进行采样 (图 1a), 共采集 7 个表层水样, 图 $1 \mathrm{~b}$ 藻华面积来自江苏环境监测网太湖蓝藻遥感 监测数据 ( 2017 年 11 月至 2018 年 3 月无记录,数据来源于 http://www.jsem.net.cn/mrygyt/thlz/) ; 月平均温 度来自无锡市气象局统计信息. 由于温度是影响藻华暴发的一个关键因素 ${ }^{[41]}$, 藻华多暴发在平均 $20^{\circ} \mathrm{C}$ 左右 的月份. 根据太湖蓝藻遥感监测数据 ${ }^{[39]}, 4-10$ 月是藻华暴发期, 11 月一次年 3 月是藻华消退期 (图 $1 \mathrm{~b}$ ). 因 此,本研究中, 2017 年 5 月、 6 月、 8 月和 2018 年 5 月采集的水样属于藻华暴发期,而 2017 年 11 月、2018 年 1 月和 3 月采集的水样属于消退期.
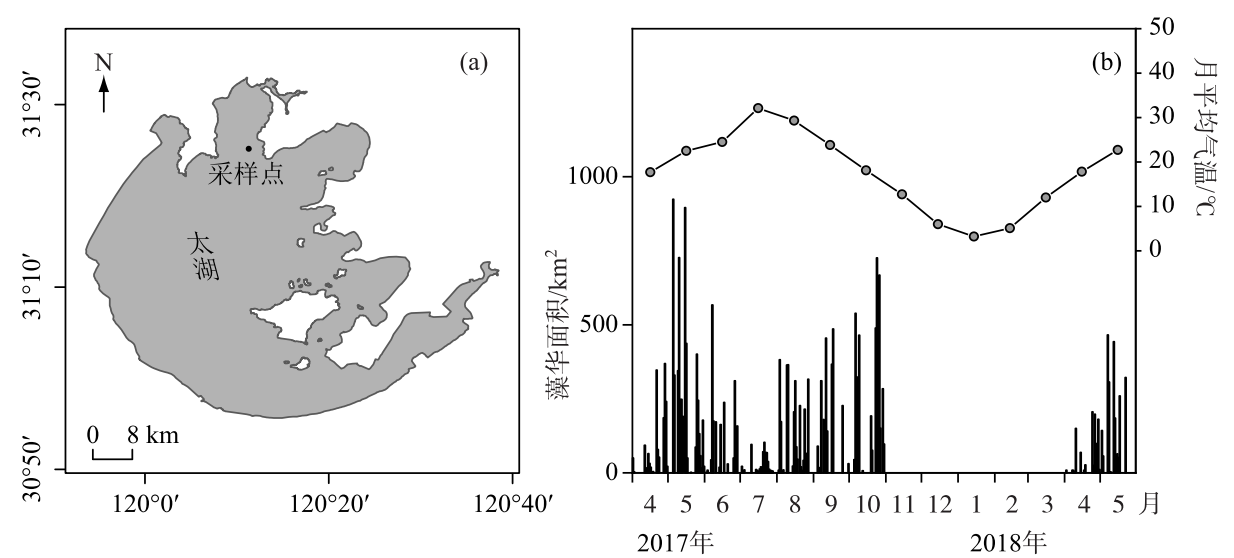

图 1 太湖梅梁湾采样点 (a) 及 2017 年 4 月- 2018 年 5 月太湖藻华面积及月平均温度 (b)

Fig.1 Sampling site in Meiliang Bay of Lake Taihu (a); algal bloom area of Lake Taihu and monthly average temperature from April 2017 to May 2018 (b)

利用采水器在水下 $0.5 \mathrm{~m}$ 内采集水样, 随后避光低温保存, 当天迅速带回实验室分析. 然后依次利用 0.7 $\mu \mathrm{m}$ 预灼烧的玻璃纤维滤纸 (Whatman) 和 $0.2 \mu \mathrm{m}$ 的聚碳酸酯滤膜 (Millipore) 进行过滤, 过滤后水样使用优 级纯盐酸将 $\mathrm{pH}$ 值调节为 2 , 在 $4^{\circ} \mathrm{C}$ 避光条件下保存并尽快进行后续分析.

预酸化的过滤水样可以直接使用高温催化氧化溶解态有机分析仪 (TOC-L analyzer, Shimadzu) 测定溶 解性有机碳 (DOC) 和溶解性总氮 (TDN) 浓度. DOC 和 TDN 的测试误差小于 $2 \%$. 碳同位素和 FT-ICR MS 分 析需要较高浓度的 DOM, 因此, 我们利用固相萃取 (SPE) 进行富集浓缩 ${ }^{[4]}$. 具体而言, 取 $800 \mathrm{~mL}$ 预酸化的 过滤水样, 调节流速 $5 \mathrm{~mL} / \mathrm{min}$ 通过 $500 \mathrm{mg}$ 的 Agilent Bond Elut PPL 固相萃取柱, 再用 $\mathrm{pH}$ 值为 2 的水溶液冲 洗固相萃取柱, 保证去除无机盐之后, 用高纯氮气将固相萃取柱吹干. 之后用约 $4 \mathrm{~mL}$ 甲醇溶液洗脱已经吹 干的固相萃取柱,获得浓缩的 SPE-DOM.

之后根据样品的 DOC 浓度和萃取过程的回收率 (以 $50 \%$ 计 $)^{[9]}$ 进行换算, 取有机碳含量约为 $30 \sim 50 \mathrm{ng}$ 的 SPE-DOM 样品置于锡杯, 低温蒸发去除甲醇之后, 通过 Thermo-Fisher MAT 253 气相色谱一同位素质谱仪 (GC-IRMS) 测得 SPE-DOM 的碳同位素值 $\left(\delta^{13} \mathrm{C}_{\mathrm{DoM}}\right.$ ), 每个样品重复测试 2 3 次, 保证标准偏差小于 $0.2 \%$, 文章使用数据为所测样品的平均值 ${ }^{[32]}$. 同时, 将 SPE-DOM 样品调至浓度为 $50 \mathrm{mg} / \mathrm{L}$ 后, 利用中国石油大学 重质油国家重点实验室的 9.4T 型 Apex-Ultra X FT-ICR MS 在负离子电喷雾 (electron spray ionization, ESI) 模 式下进行测试分析, 质量采集范围为 200 800 Da, 质谱图叠加次数为 128 次, 所有信噪比大于 4 且检测误差 小于 $1 \mathrm{ppm}$ 的质谱峰被挑选并解析为分子式. 针对解析出的分子信息, 目前已有多位研究者建立了一系列 标准来区分不同分子属性的 DOM 分子类别 ${ }^{[27-29]}$. 例如, Koch 和 Dittmar ${ }^{[28]}$ 提出芳香度指数 (aromaticity index, $A I$ ) 以对 FT-ICR MS 检测出的化合物进行分类(公式 (1)): $A I$ 值大于 0.5 的化合物为芳香族化合物, 
大于 0.67 的化合物为聚合芳香族化合物. Koch 和 Dittmar ${ }^{[31]}$ 同时考虑到 $50 \%$ 的氧原子通常存在于羰基官能 团的假设, 提出了修正的芳香度指数 $\left(A I_{\bmod }\right.$ ) (公式 $(2)$ ). 结合 $\mathrm{H} / \mathrm{C}$ 比值以及 $A I$, 可以对 FT-ICR MS 检测到 的分子进行更细致的分类 ${ }^{[33]}$ : 当分子 $1.5 \leqslant \mathrm{H} / \mathrm{C} \leqslant 2.0$ 时, 定义为脂肪族类化合物; 当 $\mathrm{H} / \mathrm{C}<1.5$ 且 $A I \leqslant 0.50$ 时, 定义为高不饱和类化合物; 当 $0.5<A I \leqslant 0.66$ 时, 定义为类多酚类化合物; 当 $A I>0.66$ 时, 定义为多环芳香 族化合物. 根据等效双键数 (double-bond equivalent, $D B E$ ) (公式 (3)) 与 $\mathrm{C} 、 \mathrm{H} 、 \mathrm{O}$ 元素的比值 ( $D B E / \mathrm{C}$ 比值在 $0.30 \sim 0.68$ 之间, $D B E / \mathrm{H}$ 比值在 $0.20 \sim 0.95$ 之间, $D B E / \mathrm{O}$ 比值在 $0.77 \sim 1.75$ 之间), 可以确定富羧酸脂环类化 合物 ( carboxyl-rich alicyclic molecules, CRAM) ——类广泛分布于 DOM 中的惰性分子 ${ }^{[13]}$.

$$
\begin{gathered}
A I=\frac{1+C-O-S-0.5 H}{C-O-S-N} \\
A I_{\bmod }=\frac{1+C-0.5 O-S-0.5 H}{C-0.5 O-S-N} \\
D B E=\frac{2+2 C-H+N}{2}
\end{gathered}
$$

\section{2 结果与分析}

\section{$2.1 \mathrm{DOC} 、 \mathrm{TDN}$ 和 $\delta^{13} \mathrm{C}_{\mathrm{DOM}}$}

2017 年 5 月至 2018 年 5 月采集的表层水体的 DOC 浓度波动范围为 $2.95 \sim 4.45 \mathrm{mg} / \mathrm{L}$, 平均值为 3.60 $\mathrm{mg} / \mathrm{L}$, 最高值出现在 2017 年 8 月, 最低值出现在 2018 年 1 月 (图 $2 \mathrm{~b}$ ). 样品的 $\delta^{13} \mathrm{C}_{\mathrm{Dom}}$ 波动范围为 $-28.2 \%$ $-24.4 \%$, 平均值为 $-27.1 \%$, 最高值出现在 2017 年 8 月 (偏正), 最低值出现在 2017 年 11 月 (图 2c). 样品的 $\mathrm{TDN}$ 浓度波动范围为 $0.42 \sim 3.88 \mathrm{mg} / \mathrm{L}$, 平均值为 $1.80 \mathrm{mg} / \mathrm{L}$, 最高值出现在 2018 年 3 月, 最低值出现在 2017 年 5 月 (图 2i). 因此, 藻华暴发期表现为高的 DOC 浓度和低的 TDN 浓度, 藻华消退期表现为低的 DOC 浓度 和高的 TDN 浓度. 这说明 DOC 主要受藻类有机质输人增加的影响, 而 TDN 可能受外源冬季污水输人以及 内源微生物矿化、分解与反硝化作用减少的影响 ${ }^{[45]}$.
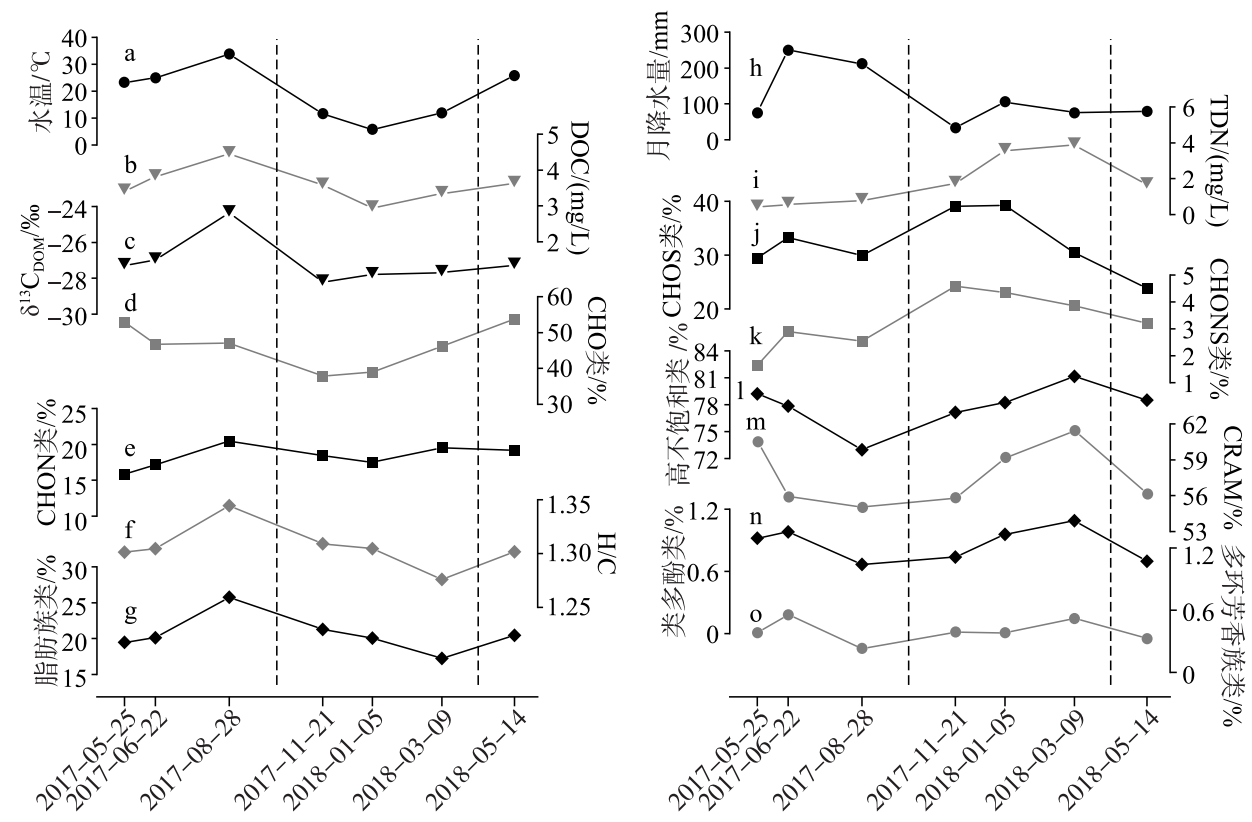

图 2 太湖梅梁湾 2017 年 5 月至 2018 年 5 月表层水体 DOM 分子参数

(虚线为藻华暴发期与消退期样品界线)

Fig. 2 Molecular parameters of surface DOM in Meiliang Bay of Lake Taihu

( The dotted line is the boundary between the algal blooming and disappearance period) 


\section{$2.2 \mathrm{DOM}$ 分子组成}

通过 FT-ICR MS 分析, 每个 DOM 样品可以解析出个数 4000 8000 不等、质荷比 $(\mathrm{m} / \mathrm{z})$ 分布在 $200 \sim 800$ 的有机化合物分子式(图 3). DOM 分子通过强度加权平均计算得到的 $\mathrm{H} / \mathrm{C}$ 比值波动范围为 $1.28 \sim 1.34$, $\mathrm{O} / \mathrm{C}$ 比值波动范围为 $0.45 \sim 0.47, A I_{\text {mod }}$ 波动范围为 $0.21 \sim 0.24$. 从时间序列上看, $\mathrm{H} / \mathrm{C}$ 比值在暴发期相对增 加, $A I_{\bmod }$ 在消退期相对增加, $\mathrm{O} / \mathrm{C}$ 比值变化趋势不明显 (表 1 ).

根据元素组成 DOM 可分为四类化合物: 只含有碳、氢、氧的 CHO 类化合物; 只含有碳、氢、氧、硫的 CHOS 类化合物; 只含有碳、氢、氧、氮的 CHON 类化合物; 含有碳、氢、氧、氮、硫的 CHONS 类化合物. 总体而 言, CHO 类化合物在 DOM 中占比最多, 占所有类别分子强度的 $37.9 \% \sim 53.0 \%$, 其次为 CHOS 类化合物 $(23.8 \% \sim 39.2 \%) 、 \mathrm{CHON}$ 类化合物 ( $15.9 \% \sim 20.5 \%) 、 \mathrm{CHONS}$ 类化合物 $(1.7 \% \sim 4.6 \%)$. 从时间序列上看, $\mathrm{CHO}$ 类化合物占比在藻华暴发期相对增加, 而 CHOS、CHONS 类化合物占比在藻华消退期相对增加, CHON 类化合物变化不明显 (图 $2 \mathrm{~d}, 2 \mathrm{e}, 2 \mathrm{j}, 2 \mathrm{k}$ ).

除根据元素组成进行 DOM 元素组成分类外, 我们还按照化合物的分子结构进行了分类统计 ${ }^{\left[{ }^{[3]}\right.}$. 其中, 高不饱和类化合物在 DOM 中占比最多 $(73.0 \% \sim 81.1 \%)$; CRAM 化合物为主要结构组分 $(55.0 \% \sim 61.4 \%)$. 其次为脂肪族类化合物 $(17.2 \% \sim 25.8 \%)$; 类多酚类化合物 $(0.7 \% \sim 1.1 \%)$; 多环芳香族类化合物 $(0.2 \% \sim$ $0.6 \%)$. 从时间序列上看, 脂肪族类化合物在暴发期相对增加, 而高不饱和类化合物和 CRAM 化合物在消退 期相对增加,类多酚类化合物和多环芳香族类化合物占比相对稳定 (图 $2 \mathrm{~g}, 2 \mathrm{l}, 2 \mathrm{~m}, 2 \mathrm{n}, 2 \mathrm{o}$ ).

表 1 太湖梅梁湾 2017 年 5 月- 2018 年 5 月基于 FT-ICR MS 分析的 DOM 分子参数特征

Tab.1 Molecular characteristics of DOM based on FT-ICR MS analysis in Meiliang Bay of Lake Taihu collected from May 2017 to May 2018

\begin{tabular}{|c|c|c|c|c|c|c|c|c|c|}
\hline $\begin{array}{l}\text { 采样 } \\
\text { 日期 }\end{array}$ & $\begin{array}{c}2017- \\
05-25^{\text {a }}\end{array}$ & $\begin{array}{c}2017- \\
06-22^{\mathrm{a}}\end{array}$ & $\begin{array}{c}2017- \\
08-28^{\mathrm{a}}\end{array}$ & $\begin{array}{l}2017- \\
11-21\end{array}$ & $\begin{array}{l}2018- \\
01-05\end{array}$ & $\begin{array}{l}2018- \\
03-09\end{array}$ & $\begin{array}{c}2018- \\
05-14^{\mathrm{a}}\end{array}$ & $\begin{array}{l}\text { 暴发期 } \\
\text { 平均值 }\end{array}$ & $\begin{array}{l}\text { 消退期 } \\
\text { 平均值 }\end{array}$ \\
\hline 分子数 & 4213 & 6054 & 7387 & 7831 & 7718 & 7484 & 6978 & 6158 & 7678 \\
\hline $\mathrm{H} / \mathrm{C}_{\mathrm{W}}^{\mathrm{b}}$ & 1.301 & 1.304 & 1.344 & 1.309 & 1.305 & 1.276 & 1.302 & 1.313 & 1.297 \\
\hline $\mathrm{O} / \mathrm{C}_{\mathrm{w}}^{\mathrm{b}}$ & 0.456 & 0.468 & 0.453 & 0.467 & 0.449 & 0.457 & 0.474 & 0.463 & 0.458 \\
\hline$A I_{\bmod }^{\mathrm{b}}$ & 0.229 & 0.220 & 0.209 & 0.213 & 0.222 & 0.240 & 0.218 & 0.219 & 0.225 \\
\hline $\mathrm{CHO}$ 类化合物强度占比/\% & 53.0 & 46.7 & 47.1 & 37.9 & 38.9 & 46.1 & 53.8 & 50.2 & 41.0 \\
\hline CHOS 类化合物强度占比 $/ \%$ & 29.5 & 33.3 & 29.9 & 39.1 & 39.2 & 30.5 & 23.8 & 29.1 & 36.3 \\
\hline $\mathrm{CHON}$ 类化合物强度占比 $/ \%$ & 15.9 & 17.1 & 20.5 & 18.4 & 17.5 & 19.6 & 19.1 & 18.2 & 18.5 \\
\hline CHONS 类化合物强度占比 $/ \%$ & 1.7 & 2.9 & 2.6 & 4.6 & 4.3 & 3.9 & 3.2 & 2.6 & 4.3 \\
\hline 脂肪族类化合物强度占比/\% & 19.5 & 20.1 & 25.8 & 21.3 & 20.1 & 17.2 & 20.5 & 21.5 & 19.5 \\
\hline 高不饱和类化合物强度占比/\% & 79.2 & 77.8 & 73.0 & 77.1 & 78.2 & 81.1 & 78.5 & 77.1 & 78.8 \\
\hline 类多酚类化合物强度占比/\% & 0.9 & 1.0 & 0.7 & 0.7 & 1.0 & 1.1 & 0.7 & 0.8 & 0.9 \\
\hline 多环芳香族化合物强度占比/\% & 0.4 & 0.6 & 0.2 & 0.4 & 0.4 & 0.5 & 0.3 & 0.4 & 0.4 \\
\hline CRAM 强度占比/\% & 60.5 & 55.9 & 55.0 & 55.8 & 59.2 & 61.4 & 56.2 & 56.9 & 58.8 \\
\hline
\end{tabular}

$\mathrm{a}$ : 藻华暴发时期; $\mathrm{b}$ :强度加权平均值.

\section{3 讨论}

\section{1 太湖藻华暴发期 DOM 分子特征}

藻华暴发期 (2017 年 5 月、 6 月、 8 月和 2018 年 5 月) 太湖梅梁湾表层水体的 DOC 浓度高, $\delta^{13} \mathrm{C}_{\mathrm{DOM}}$ 值 $(-27.3 \% 0 \sim-24.4 \% 0)$ 指示 DOM 为湖泊自生和陆源输入的混合来源 ${ }^{[46-47]}$, CHO 类化合物占比高, H/C 比值 高, 脂肪族类化合物占比高. 脂肪族类化合物由类脂质、类多肽、氨基糖等分子组成, 是易为微生物利用的活 性分子, 具有高度的生物可利用性并且能够被微生物快速利用 ${ }^{[48-50]}$. 因此, 藻华暴发期间表层水体 DOM 主 要表现为较高的 DOC 浓度、较多的自生物质来源和较强的生物活性. 尽管太湖的夏季降水量较大 (图 $2 \mathrm{~h}$ ), 在增加陆源 DOM 输人的同时, 也缩短了水体滞留时间, 会减缓藻华暴发导致的脂肪族类化合物相对含量的 


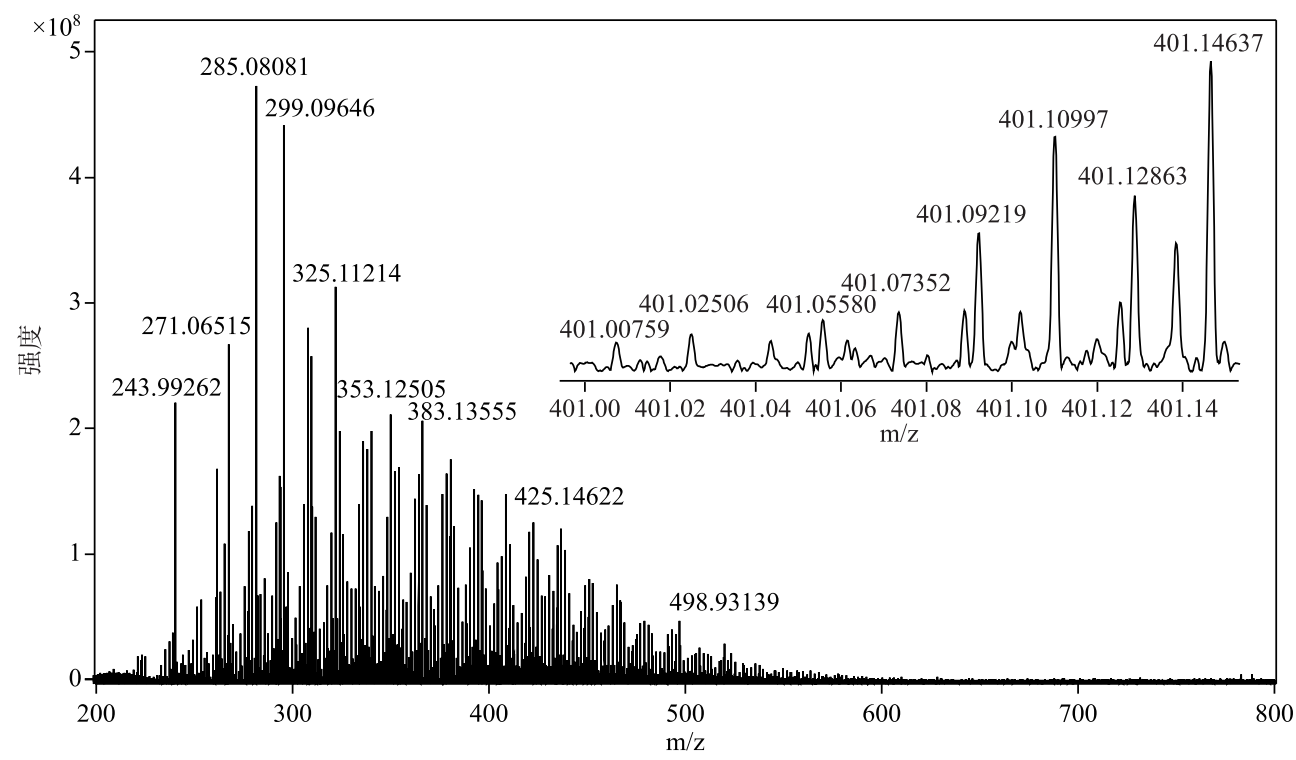

图 3 典型太湖梅梁湾 DOM 的 FT-ICR MS 质谱图 (2017 年 6 月为例)

Fig.3 FT-ICR MS mass spectra of DOM from Meiliang Bay of Lake Taihu ( sample collected on June, 2017 as an example)

增加 ${ }^{[33,51]}$. 但是, 我们的结果表明, 在富营养化藻华的背景下, 降雨对脂肪族类化合物相对含量的影响属于 次要因素. 同时, 藻华暴发期脂肪族类化合物的大量输人, 其效应较为复杂, 表现为总细菌丰度升高 ${ }^{[52]}$, 但 细菌群落多样性下降 ${ }^{[42,53]}$. 尽管活性 DOM 经过微生物代谢之后转变为惰性结构 ${ }^{[12]}$, 但在藻华暴发期脂肪 族类化合物大量输人的背景下, 该作用属于次要因素. 因此, 藻华暴发期水体 DOM 在含量上表现为 DOC 浓 度升高,在分子组成上表现为 $\mathrm{CHO}$ 类化合物、以脂肪族类化合物为代表的活性组分占比增加.

从藻华暴发初期 (2017 年 5 月) 到藻华暴发强烈期 (2017 年 8 月, 基于 DOC 浓度判断, 图 2b), 随着藻华 的持续发生和加剧, 浮游藻类大量增殖, 活体蓝藻细胞通过生物活动分泌释放的胞内代谢产物包含脂肪族 类化合物 ${ }^{[42]}$, 超过微生物的降解量, 导致 DOM 库中的活性组分含量上升. 这直观的表现为 2017 年 8 月水体 DOC 浓度最高、 $\delta^{13} \mathrm{C}_{\mathrm{DOM}}$ 值偏正约 3\%o $(-24.4 \%$ vs $-27.3 \%$ )、 $\mathrm{H} / \mathrm{C}$ 比值最高、脂肪族类化合物占比最高. 但 是, 藻华暴发强烈期水体的 CHO 类化合物在 DOM 中占比, 相较于藻华暴发初期并没有明显变化, 说明仅仅 依靠元素组成的分类方式, 并不能很好的表征持续性藻华导致活性组分增加的过程. 总体来看, 藻类释放的 DOM 与太湖水体经过了初步降解的 DOM 库在分子组成的大类上有一定的相似性, 但是化学性质更为活性.

\section{2 太湖藻华消退期 DOM 分子特征}

藻华消退期表层水体 (2017 年 11 月- 2018 年 3 月) 相较于藻华暴发期 DOC 浓度降低, DOM 在分子组 成也上发生了变化, 主要表现为 $\mathrm{H} / \mathrm{C}$ 比值降低、芳香度增加 ( 表 1); $\mathrm{CHO}$ 类化合物占比降低、CHOS、CHONS 类化合物占比增加; 脂肪族类化合物占比降低、高不饱和类化合物和 CRAM 化合物占比增加 (图 2). 其中 CHOS、CHONS 类化合物一部分来源于 CHO 化合物通过与硫化物反应形成类木质素的 CHOS 化合物, 另一 部分源于人类活动产生的表面活性剂化合物, 如磺基苯基羧酸类化合物 ${ }^{[54]}$. 高不饱和类化合物主要为酚类 结构化合物, 可能来源于木质素降解的中间产物 ${ }^{[55]}$; CRAM 化合物被认为是经微生物代谢和光降解等一系 列转化之后形成的一类惰性结构化合物 ${ }^{[13,56]}$. 类多酚类化合物主要为多酚类化合物和带有脂肪链的多环 芳香族化合物 ${ }^{[28]}$, 主要来源于维管植物 ${ }^{[33]}$; 多环芳香族化合物主要来源于燃烧物 质 ${ }^{[57]}$. 上述变化均表明, 藻华消退期主要表现为活性组分的减少和惰性组分的增加.

在藻华消退期间, 藻类进人休眠状态后沉人湖底, 其对表层水体 DOM 的直接影响大幅下降. 因此, 有机 质降解可能是藻华消退期表层水体 DOM 分子组成变化的最重要过程. 伴随多肽类、糖类等活性组分优先降 
解, 惰性组分相对耐降解而得以留存于 DOM 库内, 成为 DOM 的主要贡献者. 异养微生物作用也促进了藻类 有机质转化为 CRAM 等惰性分子 ${ }^{[9]}$. 同时, 冬季风浪扰动等水动力条件的增强,会导致部分湖底的藻类残体 和沉积物上泛, 可能也带来了一部分惰性的有机质. 除此之外, 尽管消退期的降水较弱, 但冬季本身是流域 的排污期 ${ }^{[45]}$, 可能带来了大量的难降解的陆源有机质 (如木质素等分子). 然而, $\delta^{13} \mathrm{C}_{\mathrm{DoM}}$ 除了 2017 年 8 月由 于藻华极强导致迅速偏正以外, 其他时间点的值比较相近, 基本排除了陆源输人的显著影响. 所以, 藻华消 退期表层水体惰性组分增加的主要原因可能为内源负荷的减弱,而陆源负荷的增加为次要原因.

\section{3 太湖藻华暴发期与消退期的 DOM 特征分子差异}

综上所述, 太湖水体的溶解性有机质分子组成在藻华暴发期受藻类有机质输人控制, 在消退期受藻类 有机质降解的影响. 为了进一步明晰并统计藻华暴发期与消退期的特征化合物, 我们将样品识别的所有分 子根据质谱峰强进行均一化处理, 统计各个分子在暴发期与消退期的平均相对强度, 并进行差值计算. 当分 子的相对强度差值为正数时, 指示其为暴发期的相对特征化合物 (图 4a, 蓝色), 当分子的相对强度差值为负 数时, 指示其为消退期的相对特征化合物 (图 4a, 橙色). 结合前面提到的藻华暴发一消退周期 DOM 分子的 活性和惰性特征, 可以发现暴发期 DOM 以活性小分子 $(\mathrm{m} / \mathrm{z}=200 \sim 325)$ 为特征, 消退期 DOM 以情性大分子 $(\mathrm{m} / \mathrm{z}=325 \sim 800)$ 为特征. 除此之外, 太湖藻华消退期 DOM 还包含部分小分子量的化合物 $(\mathrm{m} / \mathrm{z}=200 \sim 325)$. 这些消退期 DOM 小分子量的化合物有两种可能来源: 1 ) 未得以降解的小分子活性化合物; 2 ) 微生物降解生 成的小分子化合物 ${ }^{[14,58-60]}$. 为明晰这一问题, 我们将暴发期与消退期分子量小于 325 的化合物进行对比 (图 $4 \mathrm{c}, 4 \mathrm{~d}, 4 \mathrm{e}, 4 \mathrm{f})$, 可以发现暴发期的小分子特征化合物饱和度高 ( $\mathrm{H} / \mathrm{C}$ 比值高)、含氧量高 ( $\mathrm{O} / \mathrm{C}$ 比值高), 在 范式图中 (图 4b) 分布于类氨基糖、类糖类分子区域 ${ }^{[27]}$, 指示了新鲜分子的贡献. 相对于暴发期, 消退期的特 征分子向更高不饱和 ( H/C 比值小于 1 )、更低含氧量 ( $\mathrm{O} / \mathrm{C}$ 比值小于 0.5 ) 转移, 在范式图中分布于聚合芳香 族化合物分子区域 ${ }^{[27]}$. 导致这一变化的原因可能是由于微生物代谢消耗了高含氧的分子并产生了低含氧 的分子 ${ }^{[42]}$. 其实, 在 $\mathrm{m} / \mathrm{z}>325$ 的质量数范围, 亦可以看到太湖梅梁湾藻华暴发期到消退期的分子组成层面 上的类似变化,但由于随着分子量的增大, 所有化合物的 $\mathrm{O} / \mathrm{C}$ 比值趋于 0.4 , 而 $\mathrm{H} / \mathrm{C}$ 比值趋于 1.4(图 4c, $4 \mathrm{~d}, 4 \mathrm{e}, 4 \mathrm{f}$ ), 即使分子特征有区别也很难从范氏图中辨别出来 (图 $4 \mathrm{~b}$ ). 因此, $\mathrm{m} / \mathrm{z}=200 \sim 325$ 的分子可能更 适合表征太湖梅梁湾藻华暴发期到消退期 DOM 的分子组成变化.

本研究与 Zhang 等 ${ }^{[9]}$ 的研究结果有所差别. 具体而言,Zhang 等 ${ }^{[9]}$ 发现 2007 年 6 月到 2007 年 11 月太湖 梅梁湾水体 DOM 中, 小质量数分子和 CHON 类分子比例增加, 分子多样性提高. 我们认为, 两个研究结果的 差异可能来自样品的特殊性. 首先, Zhang 等 ${ }^{[9]}$ 分析的 2 个样品采自 2007 年, 而本研究的样品采自 $2017-$ 2018 年, 10 年的跨度可能导致水体 DOM 性质具有较大差异. 其次, 太湖流域 6 月份为梅雨季节, 降雨量较 大, 太湖水位相对较高, 对蓝藻密度具有一定的稀释作用, 一定程度上减小了蓝藻大规模聚集的程度 ${ }^{[61]}$, 因 此 6 月份不是藻华最强的时候. 而 11 月份, 蓝藻刚开始死亡和下沉, 不是藻华最弱的时候, 藻华暴发期的信 号可能还残留于 11 月份的水体中. 所以 Zhang 等 ${ }^{[9]}$ 对比 6 月和 11 月的样品, 未必能够表征藻华暴发到消退 的过程. 不仅如此, 尽管 Zhang 等 ${ }^{[9]}$ 采样点位也在梅梁湾, 但更靠近岸边, 在 6 月强降水的季节, 可能受到强 烈的陆源输人的影响, 对其是否可以确认为藻华暴发期的端元信号存在疑问. 事实上, 本研究获得的 FT-ICR MS 谱图和 Liu 等 ${ }^{[43]}$ 报道的太湖梅梁湾水体 DOM 的 FT-ICR-MS 谱图相近, 而与 Zhang 等 ${ }^{[9]}$ 报道的 FT-ICR MS 谱图差异较大. 但不论如何, 本研究结果清楚地说明了浮游藻类在太湖水体 DOM 的分子组成变化中扮演 着重要的角色. 后续仍需要开展更多太湖 DOM 分子组成时空变化的相关工作来验证本研究的结论.

\section{4 结论}

1) 在藻华暴发期, 显著增加的浮游藻类生产力, 使太湖表层水体的 DOM 从量到分子组成均发生剧烈改 变. 在含量上 DOC 浓度升高, 在分子组成上 $\mathrm{CHO}$ 类化合物、以脂肪族化合物为代表的活性组分占比增加. 在藻华消退期, 随着藻类的消亡, 藻类有机质对 DOM 直接贡献减少, 持续进行的有机质降解过程, 使得 DOC 浓度下降, CHOS、CHONS 类化合物和 CRAM 化合物等惰性分子占比增加.

2) 藻华暴发期与消退期的分子差异体现在: 暴发期以相对高饱和度和高含氧的小质量数分子为特征, 而消退期以大质量数分子和相对低饱和度和低含氧的小质量数分子为特征. 

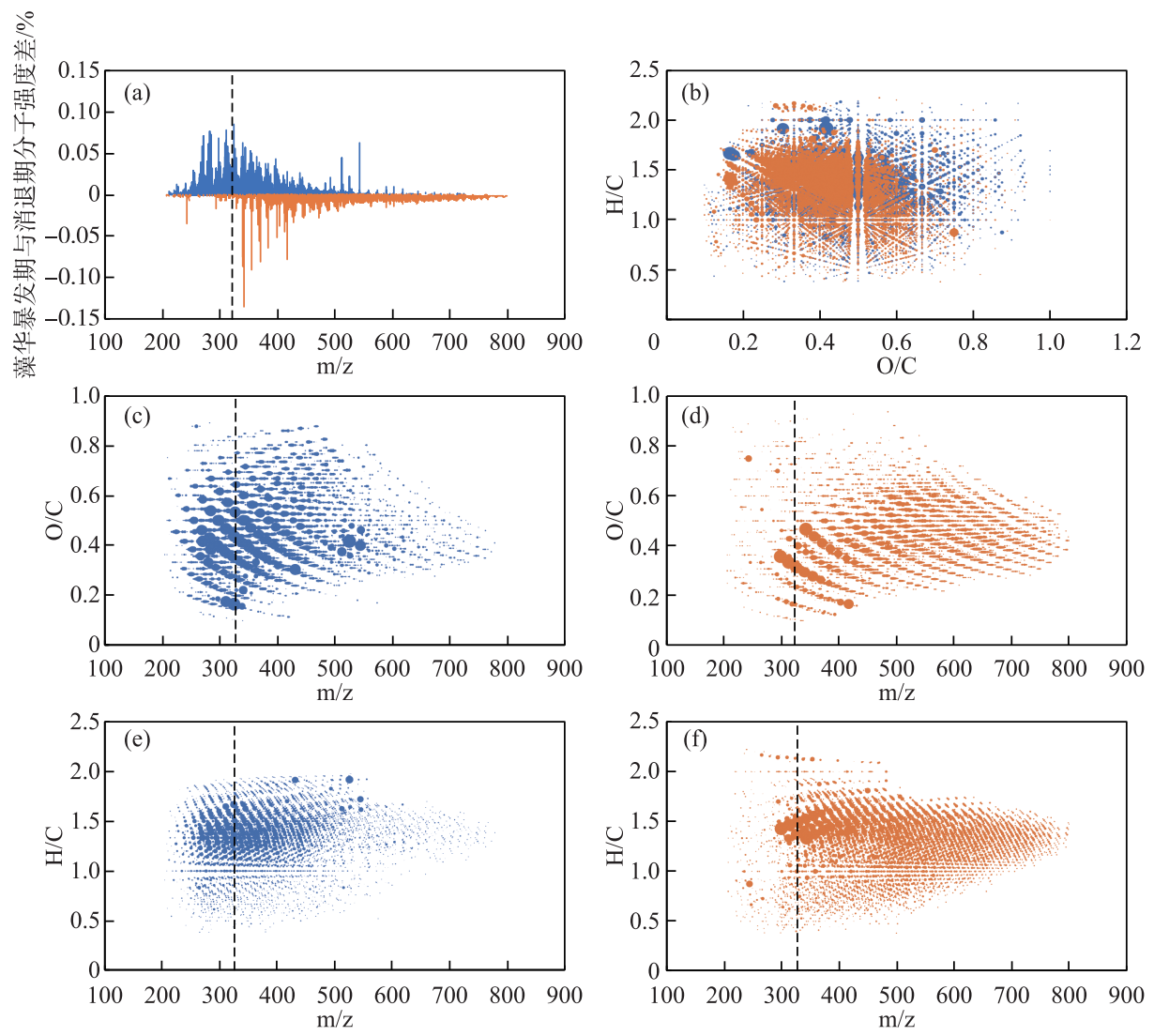

图 4 太湖梅梁湾藻华暴发期 (蓝色) 与消退期 (橙色) 表层水体 DOM 的特征分子信息 (虚线所示为 $\mathrm{m} / \mathrm{z}=325 ; \mathrm{a}$ : 藻华暴发期与消退期分子强度差; $\mathrm{b}$ : 暴发期与消退期特征分子范式图; c: 暴发期特征分子 $\mathrm{m} / \mathrm{z}$ vs $\mathrm{O} / \mathrm{C}$; d : 消退期特征分子 $\mathrm{m} / \mathrm{z}$ vs $\mathrm{O} / \mathrm{C}$; e : 暴发期特征分子 $\mathrm{m} / \mathrm{z}$ vs H/C;

$\mathrm{f}$ : 消退期特征分子 $\mathrm{m} / \mathrm{z}$ vs H/C; 图中圆形标记大小与分子强度相关)

Fig.4 Molecular characteristics of surface DOM in Meiliang Bay of Lake Taihu between the algal-blooming period (blue) and algal-disappearance period (orange) (The dotted line represents $\mathrm{m} / \mathrm{z}=325$; a: the difference of intensity between the algal-blooming period and algal-disappearance period; $\mathrm{b}$ : the Van Krevelen diagram of the algal-blooming period and the algal-disappearance period; c: $\mathrm{m} / \mathrm{z}$ vs $\mathrm{O} / \mathrm{C}$ of the algal-blooming period; $\mathrm{d}: \mathrm{m} / \mathrm{z}$ vs $\mathrm{O} / \mathrm{C}$ of the algal-disappearance period; e: $\mathrm{m} / \mathrm{z}$ vs $\mathrm{H} / \mathrm{C}$ of the algal-blooming period; $\mathrm{f}: \mathrm{m} / \mathrm{z}$ vs $\mathrm{H} / \mathrm{C}$ of the algal-disappearance period; circular marker size is related to the intensity)

3) FT-ICR MS 可以有效表征藻华暴发一消退周期内 DOM 的分子组成变化, 今后仍需结合其他分析技术 综合分析颗粒态有机质和 DOM 的变化, 为进一步分析富营养化背景下有机碳的来源构成、转化及归宿提供 科学依据.

致谢: 感谢中国科学院太湖湖泊生态系统研究站为本研究提供实验仪器设备支持.

\section{5 参考文献}

[ 1 ] Bauer JE, Cai W, Raymond PA et al. The changing carbon cycle of the coastal ocean. Nature, 2013, 504: 61-70. DOI: $10.1038 /$ nature 12857.

[ 2 ] Cole JJ, Prairie YT, Caraco NF et al. Plumbing the global carbon cycle: Integrating inland waters into the terrestrial car- 
bon budget. Ecosystems, 2007, 10(1) : 172-185. DOI: 10.1007/s10021-006-9013-8.

[ 3 ] Tranvik LJ, Downing JA, Cotner JB et al. Lakes and reservoirs as regulators of carbon cycling and climate. Limnology and Oceanography, 2009, 54(6part2) : 2298-2314. DOI: 10.4319/lo.2009.54.6_part_2.2298.

[ 4 ] Zigah PK, Minor EC, Werne JP. Radiocarbon and stable-isotope geochemistry of organic and inorganic carbon in Lake Superior. Global Biogeochemical Cycles, 2012, 26(1) : [GB1023]. DOI: 10.1029/2011GB004132.

[ 5 ] Cole JJ, Caraco NF, Kling GW et al. Carbon dioxide supersaturation in the surface waters of lakes. Science, 1994,265 (5178) : 1568-1570. DOI: 10.1126/science.265.5178.1568.

[ 6 ] Paerl HW, Paul VJ. Climate change: Links to global expansion of harmful cyanobacteria. Water Research, 2012, 46(5) : 1349-1363. DOI: $10.1016 /$ j.watres.2011.08.002.

[ 7 ] Wang CH, Jiang HL. Chemicals used for in situ immobilization to reduce the internal phosphorus loading from lake sediments for eutrophication control. Critical Reviews in Environmental Science and Technology, 2016, 46(10) : 947-997.

[ 8 ] Pacheco F, Roland F, Downing J. Eutrophication reverses whole-lake carbon budgets. Inland Waters, 2014, 4( 1): 4148. DOI: $10.5268 / \mathrm{iw}-4.1 .614$.

[ 9 ] Zhang FF, Harir M, Moritz F et al. Molecular and structural characterization of dissolved organic matter during and post cyanobacterial bloom in Taihu by combination of NMR spectroscopy and FTICR mass spectrometry. Water Research, 2014, 57: 280-294. DOI: 10.1016/j.watres.2014.02.051

[10] Canadell J, Mooney HA. Ecosystem metabolism and the global carbon cycle. Trends in Ecology \& Evolution, 1999, 14 (6) : 249. DOI: $10.1016 / \mathrm{s} 0169-5347$ (99)01601-8.

[11] Kujawinski EB, Hatcher PG, Freitas MA. High-resolution fourier transform ion cyclotron resonance mass spectrometry of humic and fulvic acids: Improvements and comparisons. Analytical Chemistry, 2002, 74(2) : 413-419.

[12] Koch BP, Witt M, Engbrodt R et al. Molecular formulae of marine and terrigenous dissolved organic matter detected by electrospray ionization Fourier transform Ion cyclotron resonance mass spectrometry. Geochimica et Cosmochimica Acta, 2005, 69(13) : 3299-3308. DOI: 10.1016/j.gca.2005.02.027.

[13] Hertkorn N, Benner R, Frommberger M et al. Characterization of a major refractory component of marine dissolved organic matter. Geochimica et Cosmochimica Acta, 2006, 70(12) : 2990-3010. DOI: 10.1016/j.gca.2006.03.021.

[14] Tremblay LB, Dittmar T, Marshall AG et al. Molecular characterization of dissolved organic matter in a North Brazilian mangrove porewater and mangrove-fringed estuaries by ultrahigh resolution Fourier Transform-Ion Cyclotron Resonance mass spectrometry and excitation/emission spectroscopy. Marine Chemistry, 2007, 105 (1/2): 15-29. DOI: 10. 1016/j. marchem.2006.12.015.

[15] Dvorski SEM, Gonsior M, Hertkorn N et al. Geochemistry of dissolved organic matter in a spatially highly resolved groundwater petroleum hydrocarbon plume cross-section. Environmental Science \& Technology, 2016, 50(11) : 5536-5546. DOI: 10.1021/acs.est.6b00849.

[16] Wetzel RG. Death, detritus, and energy flow in aquatic ecosystems. Freshwater Biology, 1995, 33(1) : 83-89.

[17] Nebbioso A, Piccolo A. Molecular characterization of dissolved organic matter (DOM) : A critical review. Analytical and Bioanalytical Chemistry, 2013, 405(1) : 109-124. DOI: 10.1007/s00216-012-6363-2.

[18] Zimmermann-Timm H. Characteristics, dynamics and importance of aggregates in rivers-an invited review. International Review of Hydrobiology, 2002, 87(2/3) : 197-240. DOI: 10.1002/1522-2632(200205) 87:2/3197: : aid-iroh197>3.0. co;2-7.

[19] Kujawinski EB, del Vecchio R, Blough NV et al. Probing molecular-level transformations of dissolved organic matter: Insights on photochemical degradation and protozoan modification of DOM from electrospray ionization Fourier transform Ion cyclotron resonance mass spectrometry. Marine Chemistry, 2004, 92(1/2/3/4) : 23-37. DOI: 10.1016/j.marchem.2004. 06.038 .

[20] Simon M, Grossart HP, Schweitzer B et al. Microbial ecology of organic aggregates in aquatic ecosystems. Aquatic Microbial Ecology, 2002, 28: 175-211. DOI: 10.3354/ame028175.

[21] Hedges JI, Keil RG, Benner R. What happens to terrestrial organic matter in the ocean? Organic Geochemistry, 1997, 27 $(5 / 6):$ 195-212. DOI: $10.1016 / \mathrm{s} 0146-6380(97) 00066-1$.

[22] Azam F, Malfatti F. Microbial structuring of marine ecosystems. Nature Reviews Microbiology, 2007, 5( 10) : $782-791$. DOI: $10.1038 /$ nrmicro1747. 
[23] Canuel EA, Hardison AK. Sources, ages, and alteration of organic matter in estuaries. Annual Review of Marine Science, 2016, 8(1) : 409-434. DOI: 10.1146/annurev-marine-122414-034058.

[24] Marshall AG. Milestones in Fourier transform Ion cyclotron resonance mass spectrometry technique development. International Journal of Mass Spectrometry, 2000, 200(1/2/3) : 331-356. DOI: 10.1016/s1387-3806(00) 00324-9.

[25] Sleighter RL, Hatcher PG. The application of electrospray ionization coupled to ultrahigh resolution mass spectrometry for the molecular characterization of natural organic matter. Journal of Mass Spectrometry, 2007, 42(5) : 559-574. DOI: 10. $1002 / j m s .1221$.

[26] Kim S, Kramer RW, Hatcher PG. Graphical method for analysis of ultrahigh-resolution broadband mass spectra of natural organic matter, the van krevelen diagram. Analytical Chemistry, 2003, 75(20) : 5336-5344. DOI: 10.1021/ac034415p.

[27] Sleighter RL, Hatcher PG. Molecular characterization of dissolved organic matter ( DOM) along a river to ocean transect of the lower Chesapeake Bay by ultrahigh resolution electrospray ionization Fourier transform Ion cyclotron resonance mass spectrometry. Marine Chemistry, 2008, 110(3/4) : 140-152. DOI: 10.1016/j.marchem.2008.04.008.

[28] Koch BP, Dittmar T. From mass to structure: An aromaticity index for high-resolution mass data of natural organic matter. Rapid Communications in Mass Spectrometry, 2006, 20(5) : 926-932. DOI: 10.1002/rcm.2386.

[29] Seidel M, Beck M, Riedel T et al. Biogeochemistry of dissolved organic matter in an anoxic intertidal creek bank. Geochimica et Cosmochimica Acta, 2014, 140: 418-434. DOI: 10.1016/j.gca.2014.05.038.

[30] Nebbioso A, Piccolo A. Molecular characterization of dissolved organic matter (DOM) : A critical review. Analytical and Bioanalytical Chemistry, 2013, 405(1) : 109-124. DOI: 10.1007/s00216-012-6363-2.

[31] Bhatia MP, Das SB, Longnecker K et al. Molecular characterization of dissolved organic matter associated with the Greenland ice sheet. Geochimica et Cosmochimica Acta, 2010, 74(13) : 3768-3784. DOI: 10.1016/j.gca.2010.03.035.

[32] Wang K, Pang Y, He C et al. Optical and molecular signatures of dissolved organic matter in Xiangxi Bay and mainstream of Three Gorges Reservoir, China: Spatial variations and environmental implications. Science of the Total Environment, 2019, 657: 1274-1284. DOI: 10.1016/j.scitotenv.2018.12.117.

[33] Kellerman AM, Dittmar T, Kothawala DN et al. Chemodiversity of dissolved organic matter in lakes driven by climate and hydrology. Nature Communications, 2014, 5: 3804. DOI: 10.1038/ncomms4804.

[34] Qin B ed. Lake Taihu, China: dynamics and environmental change. Springer Science \& Business Media, 2008.

[35] Yang GS, Ma RH, Zhang L et al. Lake status, major problems and protection strategy in China. J Lake Sci, 2010,22 (6) : 799-810. DOI: 10.18307/2010.0601. [杨桂山, 马荣华, 张路等. 中国湖泊现状及面临的重大问题与保护策 略. 湖泊科学, $2010,22(6): 799-810$.

[36] Liu X. Long-term dynamics of cyanobacteria related to environmental factors in Lake Taihu [Dissertation]. Wuhan: Huazhong University of Science \& Technology, 2012. [刘霞. 太湖蓝藻水华中长期动态及其与相关环境因子的研究 [学 位论文]. 武汉: 华中科技大学, 2012.]

[37] Ma RH, Kong FX, Duan HT et al. Spatio-temporal distribution of cyanobacteria blooms based on satellite imageries in Lake Taihu, China. J Lake Sci, 2008, 20(6) : 687-694. DOI: 10.18307/2008.0605. [马荣华, 孔繁翔, 段洪涛等. 基 于卫星遥感的太湖蓝藻水华时空分布规律认识. 湖泊科学, 2008, 20(6) : 687-694.]

[38] Gu X, Zeng Q, Mao Z et al. Water environment change over the period 2007-2016 and the strategy of fishery improve the water quality of Lake Taihu. J Lake Sci, 2019, 31(2) : 305-318. DOI: 10.18307/20190201. [谷孝鸿, 曾庆飞, 毛志刚 等. 太湖 2007-2016 十年水环境演变及“以渔改水”策略探讨. 湖泊科学, 2019, 31(2) : 305-318.]

[39] Shi K, Zhang YL, Zhang YB et al. Phenology of phytoplankton blooms in a trophic lake observed from long-term MODIS data. Environmental Science \& Technology, 2019, 53(5) : 2324-2331. DOI: 10.1021/acs.est.8b06887.

[40] Luo XC, Hang X, Cao Y et al. Dominant meteorological factors affecting cyanobacterial blooms under eutrophication in Lake Taihu. J Lake Sci, 2019, 31(5) : 1248-1258. DOI: 10.18307/2019.0512. [罗晓春, 杭釒軎云等. 太湖富营养 化条件下影响蓝藻水华的主导气象因子. 湖泊科学, 2019, 31(5): 1248-1258. ]

[41] Zhang M, Duan HT, Shi XL et al. Contributions of meteorology to the phenology of cyanobacterial blooms: Implications for future climate change. Water Research, 2012, 46(2) : 442-452. DOI: 10.1016/j.watres.2011.11.013.

[42] Bai LL, Cao CC, Wang CH et al. Toward quantitative understanding of the bioavailability of dissolved organic matter in freshwater lake during cyanobacteria blooming. Environmental Science \& Technology, 2017, 51(11) : 6018-6026. DOI: 10.1021/acs. est.7b00826. 
[43] Liu SS, He ZQ, Tang Z et al. Linking the molecular composition of autochthonous dissolved organic matter to source identification for freshwater lake ecosystems by combination of optical spectroscopy and FT-ICR-MS analysis. Science of the Total Environment, 2020, 703: 134764. DOI: 10.1016/j.scitotenv.2019.134764.

[44] Dittmar T, Koch B, Hertkorn N et al. A simple and efficient method for the solid-phase extraction of dissolved organic matter ( SPE-DOM) from seawater. Limnology and Oceanography: Methods, 2008, 6(6) : 230-235. DOI: 10.4319/lom. 2008.6.230.

[45] Wu YL, Xu H, Yang GJ et al. Progress in nitrogen pollution research in Lake Taihu. J Lake Sci, 2014, 26(1) : 19-28. DOI: 10.18307/2014.0103. [ 吴雅丽, 许海, 杨桂军等. 太湖水体氮素污染状况研究进展. 湖泊科学, 2014, 26 (1) : 19-28.]

[46] Hood E, Fellman J, Spencer RGM et al. Glaciers as a source of ancient and labile organic matter to the marine environment. Nature, 2009, 462(7276) : 1044-1047. DOI: 10.1038/nature08580.

[47] Spencer RGM, Guo WD, Raymond PA et al. Source and biolability of ancient dissolved organic matter in glacier and lake ecosystems on the Tibetan Plateau. Geochimica et Cosmochimica Acta, 2014, 142: 64-74. DOI: 10. 1016/j.gca. 2014. 08.006 .

[48] Hopkinson C, Buffam I, Hobbie J et al. Terrestrial inputs of organic matter to coastal ecosystems: An intercomparison of chemical characteristics and bioavailability. Biogeochemistry, 1998, 43(3) : 211-234. DOI: 10.1023/A:1006016030299.

[49] Søndergaard M, Middelboe M. A cross-system analysis of labile dissolved organic carbon. Marine Ecology Progress Series, 1995, 118: 283-294. DOI: 10.3354/meps118283.

[50] D'Andrilli J, Cooper WT, Foreman CM et al. An ultrahigh-resolution mass spectrometry index to estimate natural organic matter lability. Rapid Communications in Mass Spectrometry, 2015, 29(24) : 2385-2401. DOI: 10.1002/rcm.7400.

[51] Stubbins A, Spencer RGM, Chen HM et al. Illuminated darkness: Molecular signatures of Congo River Dissolved organic matter and its photochemical alteration as revealed by ultrahigh precision mass spectrometry. Limnology and Oceanography, 2010, 55(4) : 1467-1477. DOI: 10.4319/lo.2010.55.4.1467.

[52] Niu Y, Shen H, Chen J et al. Phytoplankton community succession shaping bacterioplankton community composition in Lake Taihu, China. Water Research, 2011, 45(14) : 4169-4182. DOI: 10.1016/j.watres.2011.05.022.

[53] Zhang W, Zhou YQ, Jeppesen E et al. Linking heterotrophic bacterioplankton community composition to the optical dynamics of Dissolved organic matter in a large eutrophic Chinese lake. Science of the Total Environment, 2019, 679: 136147. DOI: $10.1016 /$ j. scitotenv.2019.05.055.

[54] Melendez-Perez JJ, Martínez-Mejía MJ, Barcellos RL et al. A potential formation route for CHOS compounds in dissolved organic matter. Marine Chemistry, 2018, 202 : 67-72. DOI: 10.1016/j.marchem.2018.03.006.

[55] Stenson AC, Marshall AG, Cooper WT. Exact masses and chemical formulas of individual suwannee river fulvic acids from ultrahigh resolution electrospray ionization Fourier transform Ion cyclotron resonance mass spectra. Analytical Chemistry, 2003, 75(6) : 1275-1284. DOI: 10.1021/ac026106p.

[56] Lam B, Baer A, Alaee M et al. Major structural components in freshwater dissolved organic matter. Environmental Science \& Technology, 2007, 41(24) : 8240-8247. DOI: 10.1021/es0713072.

[57] Dittmar T, Koch BP. Thermogenic organic matter dissolved in the abyssal ocean. Marine Chemistry, 2006, 102 (3/4): 208-217. DOI: 10.1016/j.marchem.2006.04.003.

[58] Kim S, Kaplan LA, Hatcher PG. Biodegradable dissolved organic matter in a temperate and a tropical stream determined from ultra-high resolution mass spectrometry. Limnology and Oceanography, 2006, 51(2) : 1054-1063. DOI: 10.4319/lo. 2006.51.2.1054.

[59] Einsiedl F, Hertkorn N, Wolf M et al. Rapid biotic molecular transformation of fulvic acids in a Karst aquifer. Geochimica et Cosmochimica Acta, 2007, 71(22) : 5474-5482. DOI: 10.1016/j.gca.2007.09.024.

[60] Schmidt F, Elvert M, Koch BP et al. Molecular characterization of dissolved organic matter in pore water of continental shelf sediments. Geochimica et Cosmochimica Acta, 2009, 73(11) : 3337-3358. DOI: 10.1016/j.gca.2009.03.008.

[61] Liu JT, Yang YS, Gao JF et al. Characteristics of cyanobacteria bloom grading and its temporal and spatial variation in Taihu lake. Resources and Environment in the Yangtze Basin, 2011, 20(2): 156-160. [刘聚涛, 杨永生, 高俊峰等. 太 湖蓝藻水华分级及其时空变化. 长江流域资源与环境, 2011, 20(2)：156-160.] 